\title{
Activation of brown adipose tissue enhances the efficacy of caloric restriction for treatment of nonalcoholic steatohepatitis
}

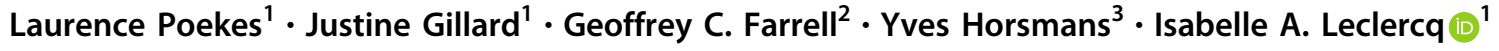

Received: 27 March 2018 / Revised: 27 July 2018 / Accepted: 2 August 2018 / Published online: 26 September 2018

(c) United States \& Canadian Academy of Pathology 2018

\begin{abstract}
Nonalcoholic steatohepatitis (NASH) is the form of nonalcoholic fatty liver disease that can evolve into cirrhosis. Lifestyle modifications achieving $10 \%$ weight loss reverse NASH, but there are no effective approved drug treatments. We previously identified defective adaptive thermogenesis as a factor contributing to metabolic syndrome and hepatic steatosis. We have now tested whether increasing nonshivering thermogenesis can improve preexisting NASH in mice. In high-fat diet-fed fozffoz mice with established NASH, treatment with $\beta 3$ AR agonist restored brown adipose tissue (BAT) function, decreased body weight, improved glucose tolerance, and reduced hepatic lipid content compared to untreated counterparts, but had no impact on liver inflammation or on nonalcoholic fatty liver disease activity score (NAS). Similarly, $\beta 3$ AR agonist did not alter liver pathology in other steatohepatitis models, including MCD diet-fed diabetic obese $d b / d b$ mice. Caloric restriction alone alleviated the hepatic inflammatory signature in $f o z / f o z$ mice. Addition of a $\beta 3 \mathrm{AR}$ agonist to mice subjected to caloric restriction enhanced weight loss and glucose tolerance, and improved liver steatosis, hepatocellular injury, and further reduced liver inflammation. These changes contributed to a significantly lower NAS score such as no (0/9) animals in this group fulfilled the criteria for NASH pathology compared to eight out of ten mice under caloric restriction alone. In conclusion, $\beta 3 \mathrm{AR}$ agonist counteracts features of the metabolic syndrome and alleviates steatosis, but does not reverse NASH. However, when coupled with weight loss therapy, BAT stimulation provides additional therapeutic advantages and reverses NASH.
\end{abstract}

\section{Introduction}

Nonalcoholic fatty liver disease (NAFLD), the hepatic manifestation of the metabolic syndrome, is now the number one cause of liver disease worldwide [1]. Its prevalence is increasing, in parallel with obesity and type 2 diabetes

Electronic supplementary material The online version of this article (https://doi.org/10.1038/s41374-018-0120-x) contains supplementary material, which is available to authorized users.

$\triangle$ Isabelle A. Leclercq

isabelle.leclercq@uclouvain.be

1 Laboratory of Hepato-Gastroenterology, Institut de Recherche Expérimentale et Clinique, Université catholique de Louvain, Brussels, Belgium

2 Liver Research Group, Australian National University Medical School at the Canberra Hospital, Canberra, ACT, Australia

3 Gastroenterology Unit, Cliniques Universitaires Saint-Luc, Université catholique de Louvain, Brussels, Belgium
[2]. The pathological spectrum of NAFLD ranges from simple steatosis to its necro-inflammatory and fibroprogressive form, nonalcoholic steatohepatitis (NASH). Having a NASH greatly increases the risks of cirrhosis, liver failure, and hepatocellular carcinoma, as well as cardiovascular events thereby increasing morbidity and mortality risks [1].

There are currently no approved therapies for NASH. Weight loss remains the most effective weapon [1, 3]. Indeed, 5\% of weight loss through lifestyle modifications has been shown to decrease liver steatosis, while $10 \%$ of weight loss significantly improved liver histology (a 2-point reduction in NAFLD activity score (NAS)), including decreased fibrosis among overweight and obese patients with NASH [4]. However, only a small proportion of patients can achieve, maintain, and sustain this level of weight loss by lifestyle interventions. Bariatric surgery could represent an alternative way to obtain long-lasting weight loss, but this is a major surgery that also requires extensive lifestyle changes [1]. Therefore, there is an interest in investigating alternative or complementary 
strategies to reverse NASH as a liver disease. Those that improve the metabolic context of NASH (prediabetes, established type 2 diabetes, and metabolic syndrome) are most attractive for an overall impact on reducing mortality from NAFLD.

There is a correlation between insulin resistance associated with obesity and low brown adipose tissue (BAT) activity. BAT is the main site of nonshivering thermogenesis in mice and humans [5]. In humans, the average BAT mass of a healthy adult is estimated to be around $50 \mathrm{~g}$, while BAT aerobic activity contributes $3-5 \%$ to basal metabolic rate $[6,7]$. As an example, in a subject with $63 \mathrm{~g}$ of supraclavicular BAT, it was calculated that if the depot was fully activated, it would burn an amount of energy equivalent to $4.1 \mathrm{~kg}$ of WAT (or $38,500 \mathrm{kcal}$ ) over the course of a year [6]. There is no consensus yet on how to measure BAT. Referring to a recent study (based on computed tomography (CT) scan and ${ }^{18} \mathrm{~F}$-labeled fluorodeoxyglucose positron emission tomography/CT $\left({ }^{18} \mathrm{~F}-\mathrm{FDG}\right.$ PET/ $\mathrm{CT})$ ), this figure may be underestimated as total BAT has been calculated to amount to $1.5 \%$ of the total body mass and $4.3 \%$ of the fat mass, with a mean volume of metabolically active BAT of $330 \mathrm{ml}$ in lean subjects [8]. The stimulation of BAT in humans can potentially increase total daily energy expenditure and conceptually represents an adjuvant target to treat obesity. Besides, increasing nonshivering thermogenesis, and thereby energy expenditure, induced beneficial effects on insulin resistance and liver steatosis in mice [9-11].

Foz/foz mice are among the few animal models that recapitulate both the metabolic context (metabolic syndrome and obesity) and hepatic characteristics observed in patients with NASH. When fed a high-fat diet (HFD), foz foz mice rapidly develop hepatic complications progressing to severe fibrosing NASH $[12,13]$. We previously reported that obesity, insulin resistance, and liver steatosis observed in $f o z / f o z$ mice were the consequences of hyperphagia and low-BAT activity, thus pointing out the role of defective BAT thermogenesis in NAFLD pathogenesis. Rescue of BAT capacity via intermittent cold exposure was able to improve glucose tolerance, decrease adiposity, and prevent development of liver steatosis [11].

A pharmacologic alternative to cold exposure as a stimulus for nonshivering thermogenesis is beta-3 adrenergic receptor ( $\beta 3 \mathrm{AR})$ stimulation. In the present study, we investigated whether increasing thermogenesis with agents such as CL-316,243 and the clinically approved mirabegron could have beneficial effects on NASH. We first demonstrated that chronic treatment effectively enhanced BAT function, corrected metabolic parameters, reduced body weight gain, and liver steatosis. In three different models of steatohepatitis (two with associated obesity and diabetes), chronic CL-316,243 treatment had no positive effects on adiposity nor liver pathology. However, when BAT stimulation was coupled with caloric restriction in $f o z / f o z$ mice with preestablished $\mathrm{NASH}$, there was, in addition to improvement of metabolic parameters, a significant decrease in steatosis, hepatocellular injury, and hepatic inflammatory signature sufficient to significantly lower NAS and reverse NASH.

\section{Methods}

\section{Animals and diets}

The fat Aussie mouse (foz/foz mouse) strain on a NOD B10 background was bred and maintained at a constant temperature of $22^{\circ} \mathrm{C}$ unless otherwise specified, and exposed at all times to a 12-h light/12-h dark cycle. At 5 weeks of age, mice were fed on a HFD ( $60 \%$ of calories from fat, $0.03 \%$ of cholesterol; Research Diets D12492) provided ad libitum.

Six-week-old C57B16J and $d b / d b$ mice were purchased from Janvier (France), and maintained in the animal facility of Université catholique de Louvain (Brussels, Belgium). After 2 weeks of acclimatization, C57B16 and $d b / d b$ mice were fed with a methionine and choline-deficient (MCD) diet.

At the time of sacrifice, blood was withdrawn by cardiac puncture and organs (liver, white adipose pads, and interscapular BAT) were dissected, weighed, snap frozen in liquid nitrogen, and stored at $-80^{\circ} \mathrm{C}$ until analyses or fixed in $4 \%$ formalin for histological analyses.

All experiments were performed according to the guidelines for humane care for laboratory animals established by the Universite catholique de Louvain in accordance with European regulations, and the study protocol was approved by the university ethics committee (2012/ UCL/MD/026; 2016/UCL/MD/003).

\section{Treatments}

For $\beta 3 \mathrm{AR}$ agonist treatment, (a) mice were implanted with an osmotic minipump (Alzet 1004, Alza, Palo Alto, CA) under $1.5 \%$ isoflurane and oxygen. The minipumps delivered either PBS or CL-316,243 (daily delivered dose was $1 \mathrm{mg} / \mathrm{kg}$ body weight) during 4 weeks together with HFD or MCD. (b) Eight-week HFD-foz/foz mice received mirabegron (or vehicle (PBS + 10\% DMSO) in controls) intraperitoneally once daily $(10 \mathrm{mg} / \mathrm{kg})$.

For caloric restriction experiment, 8-week HFD-fed foz/ $f o z$ mice were transferred in individual cages and received a restricted amount of food $(2.5 \mathrm{~g} /$ day $)$. Preliminary experiments (not shown) determined that foz/foz mice eat a mean of $3.3 \mathrm{~g}$ of $\mathrm{HFD} /$ day $(17 \mathrm{kcal} /$ day $)$, and a $25 \%$ decrease in 
calorie intake $(2.5 \mathrm{~g}$ of HFD/day or $13 \mathrm{kcal} /$ day) achieves $10 \%$ weight loss upon 2 weeks.

\section{Oral glucose tolerance test}

Glucose $(0.063 \mathrm{mg} / \mathrm{mouse})$ was administered by oral gavage to 4-h fasted animals and glucose levels were monitored on tail blood at $0,15,30,60,90,120$, and $180 \mathrm{~min}$ after the gavage using a glucometer (Accuchek). Cumulative area under the curve for blood glucose over $180 \mathrm{~min}$ was calculated.

\section{Cold challenge}

HFD-fed fozlfoz mice were transferred in cages (two mice per cage) with no bedding and maintained at $4{ }^{\circ} \mathrm{C}$ for $2 \mathrm{~h}$. Body temperature was measured every $2 \mathrm{~h}$ using an electronic thermistor equipped with a rectal probe (Bioseb thermometer TK 8851).

\section{PET/CT imaging}

A $150 \mu \mathrm{Ci}$ of ${ }^{18} \mathrm{FDG}$ (Betaplus Pharma, Brussel, Belgium) was injected into the peritoneal cavity and PET/CT scan images were acquired $1 \mathrm{~h}$ later using a Gemini PET/CT system (Philips Healthcare) on anesthetized mice. The PET and CT images were then analyzed with the PMod software. The interscapular BAT was manually delineated in three dimensions. ${ }^{18} \mathrm{~F}$-FDG BAT uptake was expressed as a percentage of injected dose per gram of BAT as described previously [11].

\section{Histology and IHC}

Five-micrometer-thick paraffin sections were stained with hematoxylin/eosin and scored for NAS by a blinded examinator according to the system devised for human NASH [39]. BAT lipid content was quantified using Visiopharm software. Uncoupling protein 1 (UCP1), macrophages, and neutrophils were detected by IHC using a primary polyclonal rabbit antimouse UCP1 Ab (Abcam) with an anti-rabbit streptavidin horseradish peroxidase-conjugated $\mathrm{Ab}$ (EnVision; Dako), or rat anti-mouse F4/80 Ab (Abd Serotec MCA497G) or rat antimouse Ly6G Ab (BD Pharmingen 551459), followed by a secondary rabbit anti-rat $\mathrm{Ab}$ and anti-rabbit EnVision. Peroxidase activity was revealed with diaminobenzidine. Quantitative measures of staining intensity and area were obtained using the Leica Tissue IA software.

\section{RNA extraction, reverse transcription, and real-time quantitative polymerase chain reaction}

cDNA was synthesized from $1 \mu \mathrm{g}$ of RNA. Gene expression was assessed by quantitative polymerase chain reaction
(PCR) with the Step One Plus device and software (Applied Biosystems, CA, USA) using SYBRGreen (Applied Biosystems). mRNA levels were normalized to that of ribosomal protein L19 (RPL19) as a reference gene.

\section{Biochemical analyses}

Serum cAMP levels were measured using a direct cAMP ELISA kit (Enzo Life Science). NRG4 protein levels in BAT were measured using an ELISA kit (MyBiosource). Total liver lipids were extracted with methanol and chloroform and quantified using the vanillin phosphoric acid reaction [14].

\section{Statistics}

All data are presented as mean \pm S.E.M. Statistical analyses were performed using a two-tailed Student's $t$ test or oneway ANOVA followed by Bonferroni's post hoc test as appropriate using Graph Pad Prism 7 software. Differences were considered significant at values of $p<0.05$.

\section{Results}

\section{HFD causes NASH and compromises BAT function in foz/foz mice}

As also reported elsewhere [12, 14, 15], foz/foz mice fed with a HFD for 12 weeks are overtly obese, hyperglycemic, and glucose intolerant compared to chow-fed (normal diet) littermates (Fig. 1a-c). They exhibit hepatomegaly (Fig. 1d), and develop steatohepatitis as indicated by elevated transaminase levels (Fig. 1e, f), and liver histology with a mean NAS score at 6.6 (range: [5-8], noting 8 is the maximum score possible) (Fig. 1g). Confirming previous data [11], while having normal baseline body temperature (Fig. 1h), the phenotype of HFDfed fozlfoz mice is associated with defective thermogenesis and poor BAT function, as assessed here by reduced uptake of ${ }^{18} \mathrm{~F}$-FDG substrate upon cold exposure evaluated in vivo by PET/CT (Fig. 1i). The obese, metabolic, thermogenic, and hepatic changes were already present after 8 weeks of HFD feeding. Noticeably, there is a worsening of the hepatic phenotype between 8 and 12 weeks with a further decreased BAT activity $(p<0.05)$ (Fig. 1i), elevation of serum transaminase levels $(p<0.001)$ (Fig. 1e, f), and aggravation of liver pathology (mean NAS score of 5.3 at 8 weeks vs. 6.6 at 12 weeks, $p<0.05$ ) (Fig. 1g).

\section{$\beta 3 A R$ agonists stimulate BAT function in HFD-fed foz/foz mice}

To test the effects of a $\beta 3 \mathrm{AR}$ agonist treatment on NASH progression, we treated foz/foz mice that had been 
Fig. 1 Under a HFD, fozlfoz mice become obese, insulin resistant, and progressively develop severe NASH. Body weight (a), fasting blood glucose levels (b), area under the glucose curve during a 180-min glucose tolerance test (c), liver weight (d), serum ALT (e), et AST (f), NAS score according to Kleiner et al. [39] (g) in foz/foz mice fed with a normal diet for 12 weeks $(n=7)$ or a HFD for $8(n=6)$ and 12 weeks $(n=10)$, baseline body temperature (h), and ${ }^{18} \mathrm{~F}$ fluorodeoxyglucose uptake by the brown adipose tissue measured by PET/scan ( $\mathrm{i}$-data are expressed as the percentage of ${ }^{18} \mathrm{~F}$-FDG injected dose per gram of BAT) in fozlfoz mice fed with a normal diet for 12 weeks $(n=5)$ or a HFD for 8 $(n=7)$ and 12 weeks $(n=5)$. Dot plots indicate values for each single mouse, the mean, and S.E.M. for each group.

One-way ANOVA and

Bonferroni correction for multiple testing. ${ }^{*} p<0.05$; ${ }^{* *} p<0.01 ;{ }^{* * *} p<0.001$
A

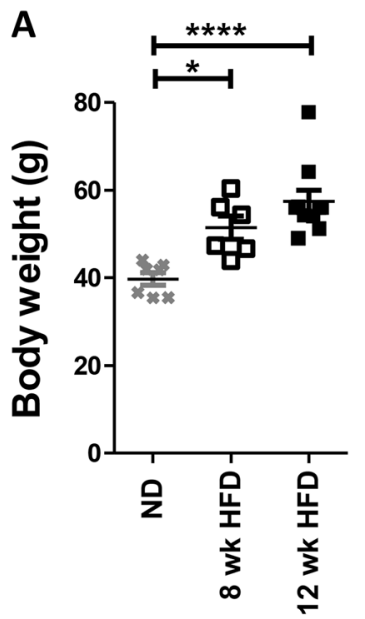

B

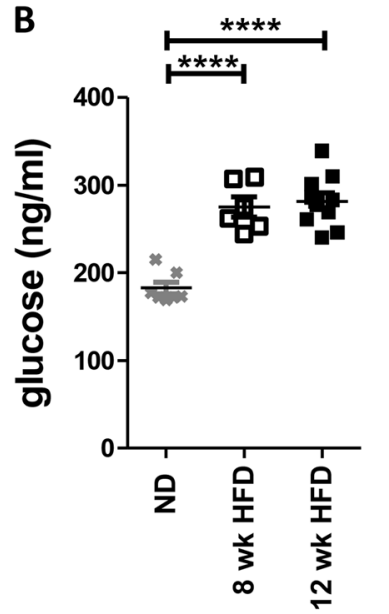

D

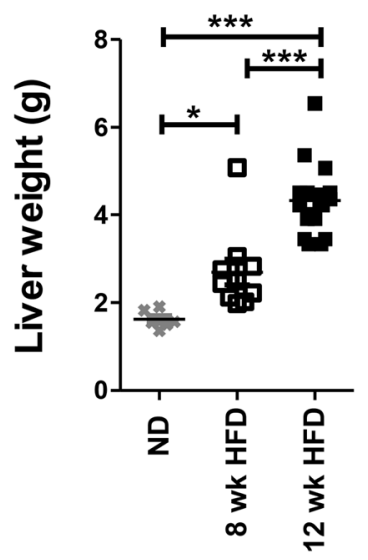

$\mathbf{E}$

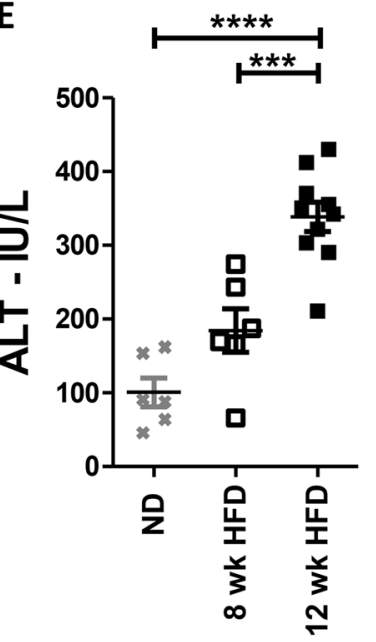

G

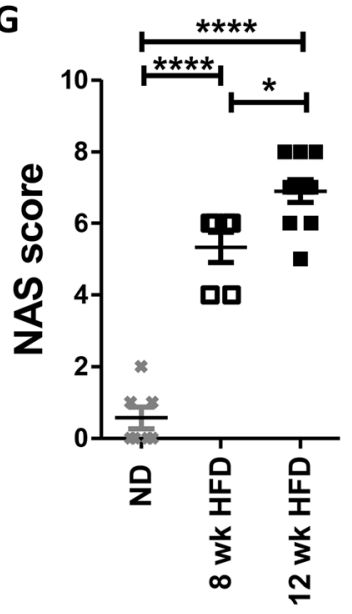

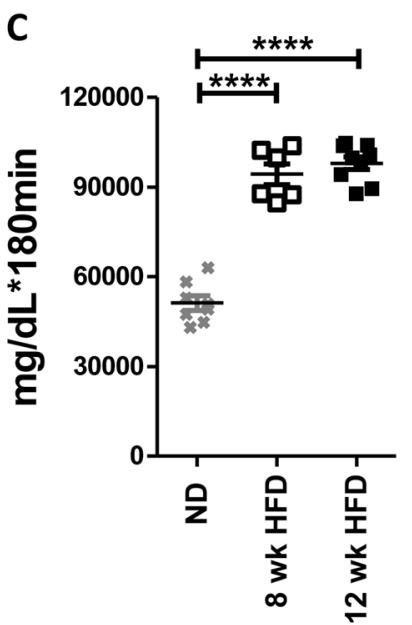

F

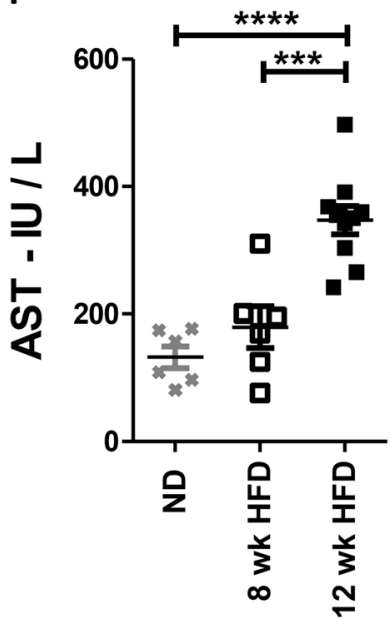

fed the HFD diet for 8 weeks with CL-316,243 (vehicle, phosphate-buffered saline (PBS) to positive controls) via an implanted minipump for a further 4 weeks while the HFD regiment continued. To assess the metabolic changes and evolution of liver pathology, we compared the $\beta 3 \mathrm{AR}$ - and PBS-treated groups with pretreatment 8-week HFD-fed mice.
I
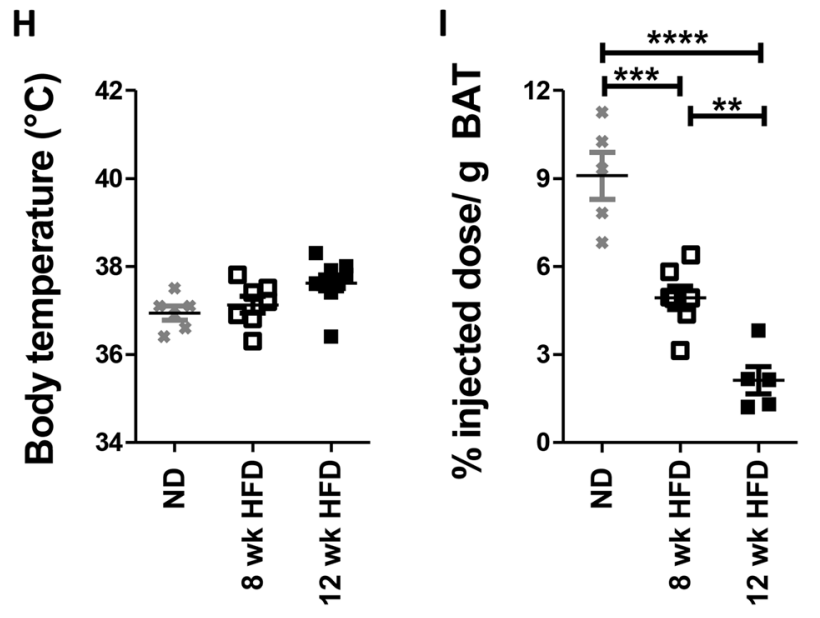

In BAT, CL-316,243 treatment increased cAMP content, the second messenger of the $\beta 3$ adrenoreceptor (Fig. 2a). This was linked to an enhanced thermogenic program (Fig. 2b), evident by induction of expression of UCP1 by a factor 10. This was associated with an increase in UCP1 protein in the tissue, as shown by IHC (Fig. 2c) and confirmed by western blot (Suppl. Figure 2). Beside UCP1, the 


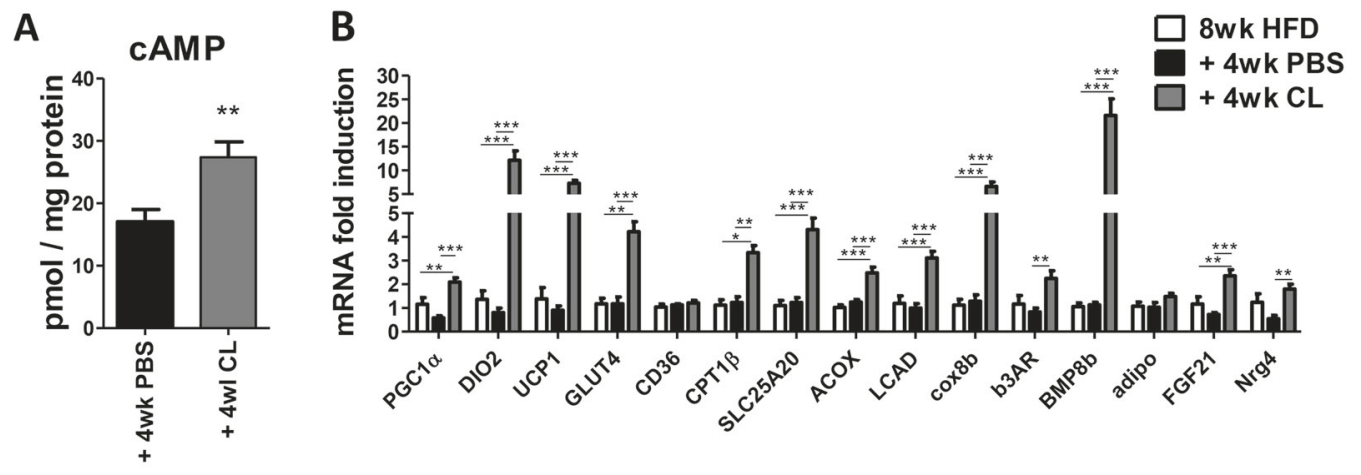

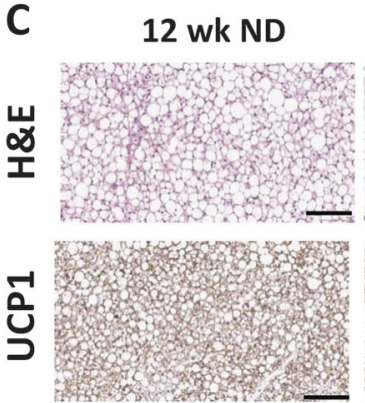
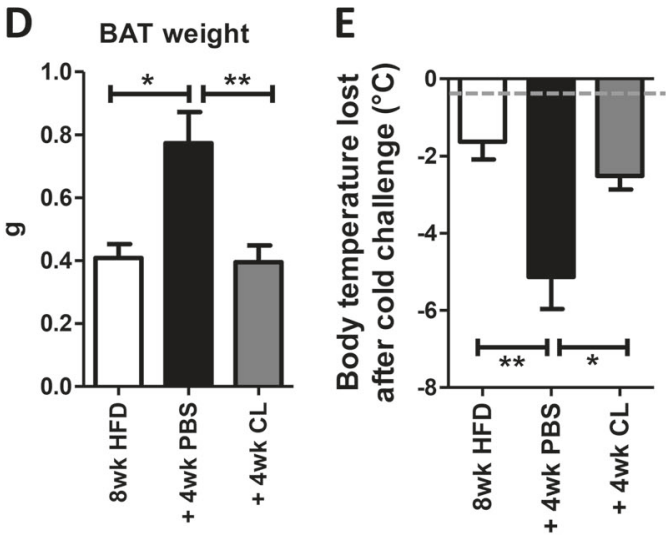

Fig. $2 \beta 3$ AR agonist CL-316,243 effectively reactivates the BAT in HFD-fed foz/foz mice. After 8 weeks of HFD (white bars, $n=6$ ), fozl foz mice were implanted with a minipump to deliver the $\beta 3 \mathrm{AR}$ agonist CL-316,243 (gray bars, $n=8$ ) or PBS (black bars, $n=8$ ) for 4 weeks, together with HFD. a cAMP content in BAT. b mRNA expression of genes related to thermogenesis, $\beta$-oxidation, and batokines in BAT of CL-316,243-treated and PBS-HFD fozlfoz mice at the end of treatment. Data are expressed as fold induction compared to values of the pretreatment (8-week HFD) group. c Hematoxylin and eosin staining (upper panels) and immunohistochemical staining of UCP1 (lower panels) in BAT (bar size, $100 \mu \mathrm{m}$ ). Fozlfoz mice on a normal diet (ND,

treatment induced the expression of other BAT genes involved in thermogenesis (PGC1 $\alpha$ and $\mathrm{DIO} 2)$, lipolysis, and in $\beta$-oxidation (CPT1B, SLC25A20, ACOX, LCAD, and COX8b) and glucose uptake (GLUT4) (Fig. 2b). Also, the expression levels of BMP8b, shown to increase BAT thermogenesis [40], as well as FGF21 and NRG4 endocrine factors known to have beneficial effects on liver metabolism $[16,17]$, increased in the BAT of CL-316,243-treated mice (Fig. 2b). The molecular signature supports increased
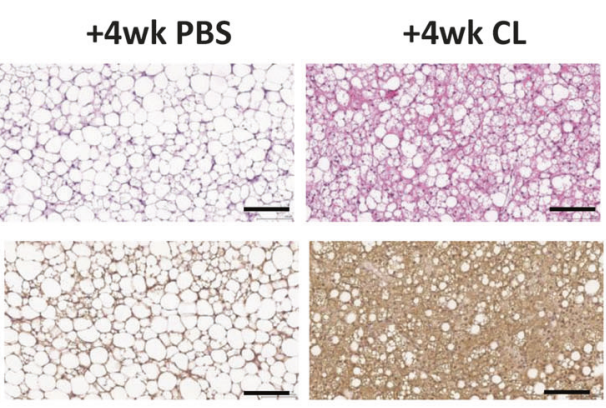

$\mathbf{F}$

$\mathbf{G}$
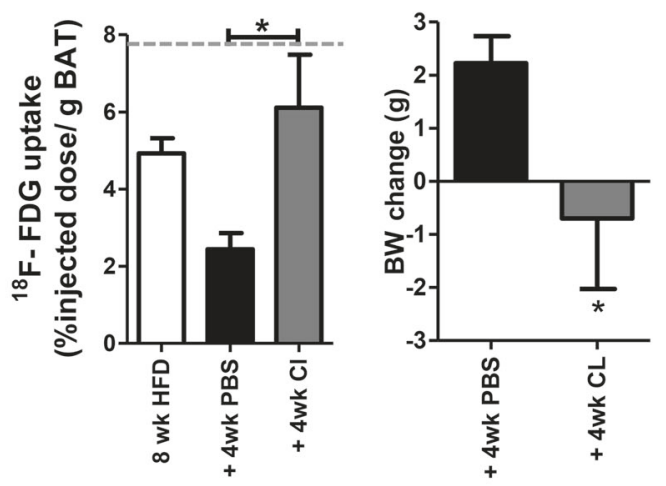

standard rodent chow) for the same 12-week duration are controls. d BAT weight. e Change in intrarectal temperature before and after a 2-h exposure at $4{ }^{\circ} \mathrm{C}$. f ${ }^{18} \mathrm{~F}$-fluorodeoxyglucose uptake by interscapular BAT measured by PET/scan in fozlfoz mice. Data are expressed as the percentage of ${ }^{18}$ F-FDG injected dose per gram of BAT. The dotted line in $\mathbf{e}$ and $\mathbf{f}$ represents values in controls (fozlfoz on the ND for 12 weeks). g Body weight change during the 4-week PBS or CL316,243 treatment. The results are expressed as mean + S.E.M. for each group. One-way ANOVA and Bonferroni correction for multiple testing. ${ }^{*} p<0.05 ;{ }^{* *} p<0.01 ;{ }^{* * *} p<0.001$

thermogenic activity and this was confirmed by a decreased weight and fat content in BAT (Fig. 2c, d). CL-316,243 treatment did not associate with changes in body temperature compared to PBS-treated counterpart (Suppl. Figure 1). The physiological functionality of BAT was tested by exposing mice at $4{ }^{\circ} \mathrm{C}$ for $2 \mathrm{~h}$. Compared to PBS counterparts that experienced a $5{ }^{\circ} \mathrm{C}$ drop of their body temperature, CL-316,243-treated HFD-fed fozlfoz mice only lost $2{ }^{\circ} \mathrm{C}$ as the pretreatment group $(p<0.05)$ (Fig. 2e). Under 

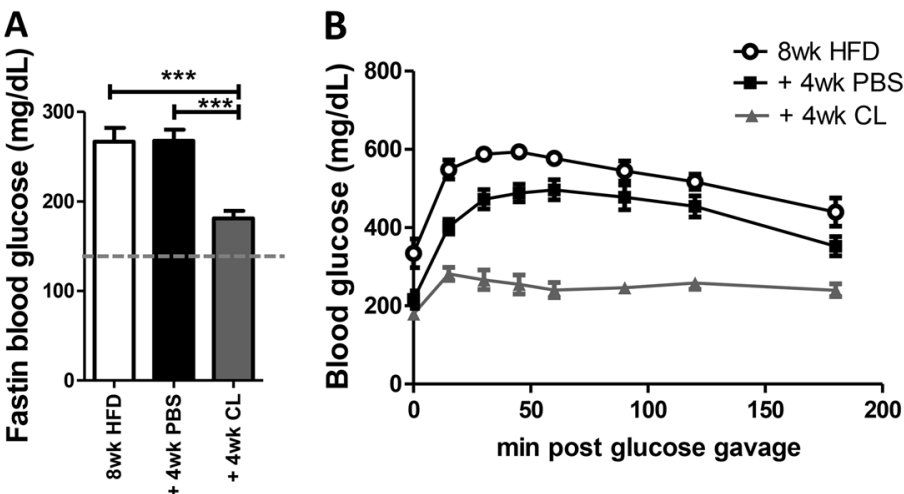

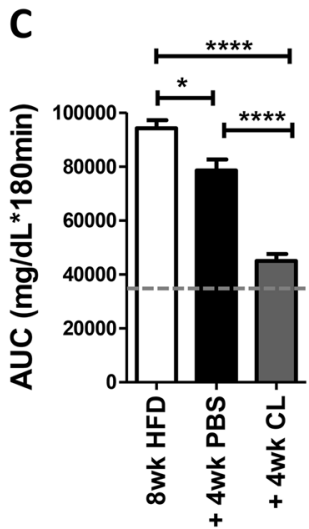

Fig. 3 33AR agonist CL-316,243 improves glucose homeostasis in HFD-fed foz/foz mice. After 8 weeks of HFD (white bars; open symbols, $n=6$ ), fozlfoz mice were implanted with a minipump to deliver the $\beta 3 \mathrm{AR}$ agonist CL-316,243 (gray bars; gray symbols, $n=8$ ) or PBS (black bars, black symbols, $n=8$ ) for 4 weeks, together with HFD. Fasting blood glucose levels (a). Oral glucose tolerance test:

these conditions, glucose uptake by BAT, measured by ${ }^{18}$ F-FDG PET, was enhanced significantly in CL-316,243treated mice (Fig. 2f). The $\beta 3 \mathrm{AR}$ agonist prevented weight gain during the treatment period (Fig. 2g), and this was not attributable to a lower food intake as CL-316,243-treated mice consumed at least as much food $(3.3 \pm 0.7 \mathrm{~g}$ of HFD/ day/mouse) as PBS-treated littermates $(2.8 \pm 0.5 \mathrm{~g}$ of HFD/ day/mouse; $p=$ not significant).

\section{B3AR agonist treatment improves metabolic syndrome, decreases hepatic fat but does not improve NASH in HFD-fed foz/foz mice}

Together with reducing body weight (Fig. 2g), CL-316,243 treatment normalized fasting blood glucose levels (Fig. 3a) and dramatically improved glucose tolerance (Fig. 3b, c). The treatment significantly reduced liver weight (Fig. 4a), steatosis, hepatic lipid content (Fig. 4b, d), and downregulated genes that mediate lipogenesis (FAS and ACC), while the expression of genes involved in $\beta$-oxidation was unaffected (Fig. 4c). We also observed a decreased TNF $\alpha$ and collagen $\alpha 1$ gene expression, but not of F4/80 and CD68, markers of inflammatory macrophages, nor of $\alpha$ SMA, a marker of hepatic stellate cell activation (Fig. 4e). Despite these positive changes at the level of gene expression, there is no effect on transaminase levels (Fig. 4f) or liver pathology, as indicated by the NAS score (Fig. 4g).

\section{B3AR agonist has no therapeutic effect on nonmetabolic steatohepatitis induced by the MCD diet}

We further tested the effect of CL-316,243 treatment and BAT activation on steatohepatitis induced by the MCD diet. blood glucose curves (b) and area under the glucose curves (c). Data are represented as mean \pm S.E.M. The dotted line in $\mathbf{a}$ and $\mathbf{c}$ represents values in controls (foz/foz on the ND for 12 weeks). Oneway ANOVA followed by post hoc Bonferroni analysis. ${ }^{*} p<0.05$; ${ }^{* *} p<0.01 ;{ }^{* * *} p<0.001$

To counterbalance the cachectic and catabolic status occurring upon MCD feeding [18], we used obese and diabetic $d b / d b$ mice fed with the MCD diet. In such a metabolic setting, $\beta 3 \mathrm{AR}$ agonist treatment enhanced BAT activity as assessed by decreased BAT weight and fat content (Suppl. Figure 3A-C), induction of UCP1 gene, and protein expression and induction of DIO2 mRNA (Suppl. Figure 3C, D). Consequent to such activation, treated MCD $d b / d b$ mice better adapted to acute cold exposure (Suppl. Figure 3B). MCD per se had a dramatic impact on glucose homeostasis, the additional effect of CL-316,243 being only marginal (Suppl. Fig. 3E). In spite of this, we saw no change in the severity of steatohepatitis with steatosis, inflammation, fibrosis, and serum transaminase elevation of similar magnitude between PBS and treated groups (Suppl. Fig. 3F-I). Thus, in this model in which steatohepatitis was induced by MCD and thus pathogenically unrelated to obesity and energy substrate overload, $\beta 3 \mathrm{AR}$ agonist administration with concomitant high-BAT activity had no protective effect on liver pathology.

\section{Coupling BAT stimulation and moderate caloric restriction improves preestablished metabolic syndrome and NASH}

A moderate (10\%) body weight is a highly effective strategy for NASH treatment $[4,19,20]$, but long-term maintenance has proven difficult in patients. BAT activation could help maintaining weight loss. To evaluate the effect of such a strategy on NASH, we subjected foz/foz mice with preestablished NASH to a moderate caloric restriction combined with a $\beta 3 \mathrm{AR}$ agonist treatment or PBS for 4 weeks and compared them with littermates fed ad libitum the HFD, treated with vehicle, and single-housed in similar 
A

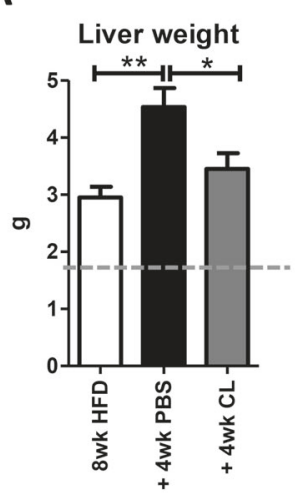

D Chow fed control

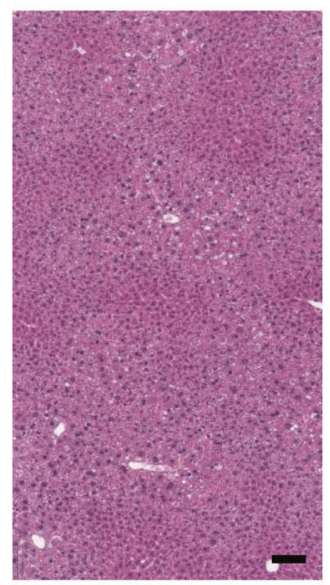

E

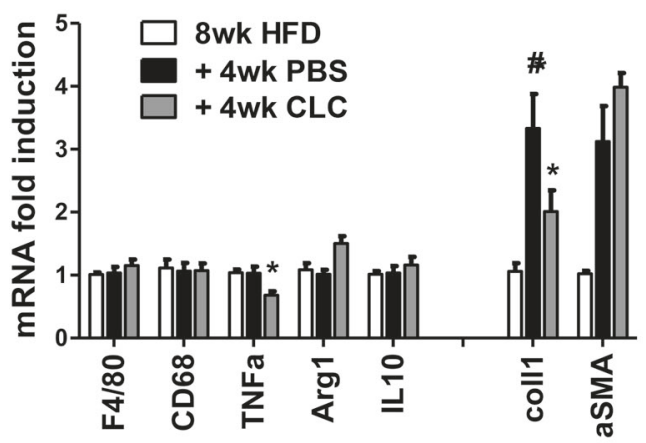

Fig. 4 33AR agonist CL-316,243 lessens steatosis in HFD-fed foz/foz mice with preestablished NASH. After 8 weeks of HFD (white bars, $n$ $=6$ ), fozlfoz mice were implanted with a minipump to deliver the $\beta 3 \mathrm{AR}$ agonist CL-316,243 (gray bars, $n=8$ ) or PBS (black bars, $n=$ 8) for 4 weeks, together with HFD. a Liver weight, (b) hepatic lipid content, and (c) hepatic mRNA expression of genes involved in lipid metabolism. Data are expressed as fold induction compared to values of the pretreatment (8-week HFD) group. d Hematoxylin and eosin staining of liver sections (original magnification: upper panels $10 \times$ and lower panels 20x; bar size: $100 \mu \mathrm{m}$ ). Foz/foz mice on a normal diet

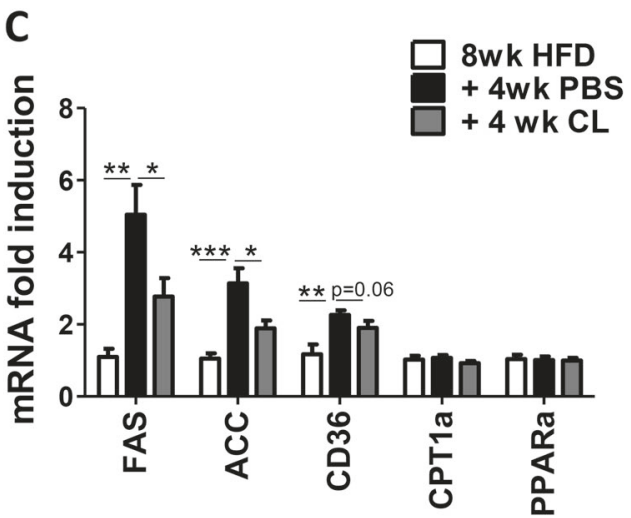

+4wk PBS
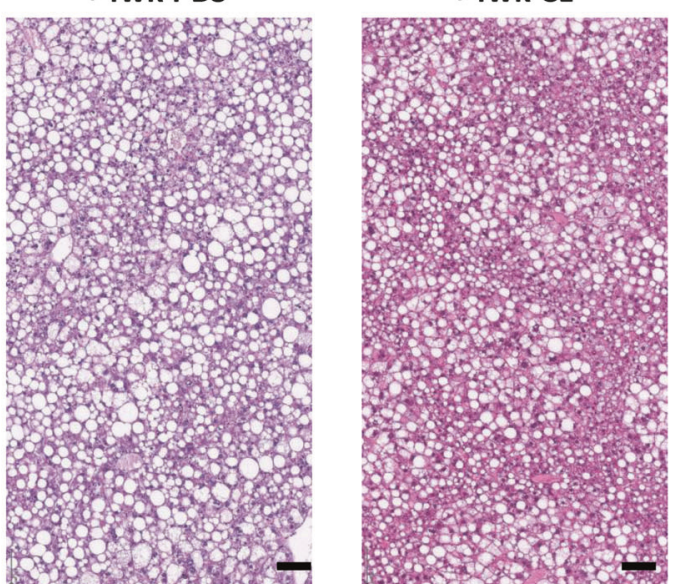

$\mathbf{F}$

G

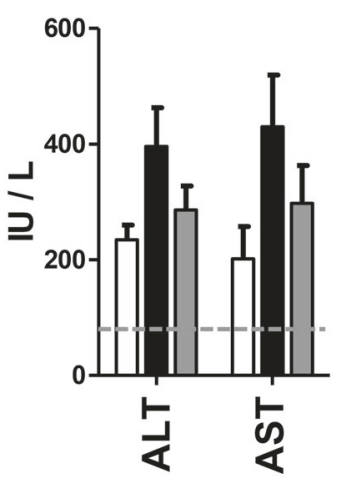

(ND, standard rodent chow) for the same 12-week duration are controls. e Hepatic mRNA expression of genes involved in inflammation and fibrosis. Data are expressed as fold induction compared to values of the pretreatment (8-week HFD) group. f Serum transaminases. Data are represented as mean \pm S.E.M. One-way ANOVA followed by post hoc Bonferroni analysis. ${ }^{*} p<0.05 ;{ }^{* *} p<0.01 ;{ }^{* * *} p<0.001$. g Histological NAS score with the mean steatosis score (white part of the bars), the ballooning score (dotted part of the bars), and the inflammatory score (hatched part of the bars). The dotted line in $\mathbf{a}, \mathbf{b}, \mathbf{f}$, and g represents values in controls (fozlfoz on the ND for 12 weeks) 
conditions. We titrated caloric restriction to obtain a $10 \%$ weight reduction in HFD-fed PBS-treated mice. $33 \mathrm{AR}$ agonist-treated mice received daily the exact same amount of food than PBS-treated mice. Because in mice with food restriction, prolonged CL-316,243 administration caused high mortality (five out of eight, not shown), we used in this experiment mirabegron, a well-tolerated and clinically approved $\beta 3 \mathrm{AR}$ agonist. At the recommended dose of $10 \mathrm{mg} / \mathrm{kg}$ once a day [21], mirabegron potently activated BAT (Suppl. Figure 4) with no observed adverse effect in chow-fed mice.

As targeted, HFD-diet-fed foz/foz mice subjected to food restriction alone lose $10 \%$ of the BW (Fig. 5a). This had no effect on BAT weight $(0.33 \pm 0.07 \mathrm{~g}$ vs. $0.39 \pm 0.04 \mathrm{~g}$ in ad libitum-fed, single-housed controls, NS), fat content (Fig. 5b), expression of UCP1, and of other thermogenic genes (Fig. 5b-d) or on tolerance to cold temperatures (Fig. 5e), on fasting blood glucose (Fig. 5f), and on glucose tolerance (Fig. 5g). Mirabegron successfully stimulated BAT and enhanced thermogenic capacities: while baseline temperature was unaffected (Suppl. Figure 5), it significantly improved cold tolerance (Fig. 5e), decreased BAT weight $(0.19 \pm 0.06$ vs. $0.33 \pm 0.07$ in PBS-treated mice, $p<0.01$ ), and fat content (Suppl. Figure 5B). Mirabegron increased UCP1 protein in BAT (Fig. 5b, c), upregulated genes involved in thermogenesis (UCP1 and DIO2), $\beta$ oxidation (CPT1B, SLC25A20, ACOX, and LCAD), and mRNA expression of batokines (BMP8b, adiponectin, FGF21, and NRG4) (Fig. 5d). Mirabegron together with food restriction amplified the weight loss to $25 \%$ compared to $10 \%$ in untreated mice receiving the exact same amount of food (Fig. 5a). Increased nonshivering thermogenesis and weight loss in mirabegron-treated mice decreased fasting blood glucose (Fig. 5f) and improved glucose tolerance (Fig. 5g) compared with mice with caloric restriction alone.

Calorie restriction alone had no significant impact on liver steatosis (Fig. 6), liver weight, fat content, or hepatic lipogenesis (Fig. 7a-c). By contrast, all those parameters decreased in mice on calorie restriction plus mirabegron treatment. Mirabegron improved steatosis (Fig. 6). Reduced liver weight (Fig. 6a) and fat content (Figs. 6 and 7b) likely resulted from the cumulative effect of reduced hepatic lipogenesis (Fig. 7c) and increased energy substrate burning in the BAT (Fig. 5b-d). Restriction of calorie intake was associated with a reduction in transaminase serum levels (Fig. 7f), and in the expression of inflammatory markers F4/ 80 and CD68 and of proinflammatory cytokines in the liver (Fig. 7e). We also observed a decreased density of F4/80+ macrophages (Fig. 6b) and Ly6G + neutrophils (Fig. 6c) in liver parenchyma, but not in the number of inflammatory foci as translated by the inflammatory score (2) similar to that of ad libitum fed mice (Fig. 7g). Superimposition of mirabegron treatment to calorie restriction further decreased liver inflammation as shown by a further reduction in the expression of TNF $\alpha$ and IL1 $\beta$ (Fig. 7e), in the amount of macrophages and recruited neutrophils (Fig. 6b, c). Mirabegron decreased transaminase levels (Fig. 7f). Noticeably, a significant lowering of hepatocyte ballooning was also observed in mirabegron-treated livers (Fig. 7d) as well as in collagen 1 expression. Cumulatively, the benefit of mirabegron treatment over food restriction alone on steatosis, ballooning and inflammation translates into a significant decrease in NAS score (mean $3.2 \pm 1$, range: [1-4]) such as none $(0 / 9)$ of the mirabegron-treated mice fulfilled the criteria for NASH vs. $5 / 5$ in ad lib fed and 5/8 in PBS-food restricted controls $(p<0.05)$ (Fig. $7 \mathrm{~g}$ ).

\section{Discussion}

In the present work, we studied the effects of BAT stimulation using a $\beta 3 \mathrm{AR}$ agonist on metabolic and hepatic complications of obesity. In foz/foz mice with a severe metabolic syndrome, low-BAT activity and progressive fibrotic NASH increasing thermogenic capacity via $\beta 3 \mathrm{AR}$ agonist treatment improved glucose homeostasis and limited ectopic fat accumulation in the liver. Moreover, when coupled with weight loss therapy, BAT stimulation provides additional therapeutic advantages: it cleared hepatic lipids, reduced hepatocellular injury and inflammation, and reversed NASH.

An increasing number of studies support that activation of the thermogenic program in the adipose tissue beneficially impacts on glucose and lipid homeostasis and insulin sensitivity, especially in the context of energy overload and obesity $[9,10,22,23]$. In the HFD-fed fozlfoz mouse model we used, low-thermogenic BAT function accompanies severe insulin resistance and morbid obesity [11]. Treatment with $\beta 3 \mathrm{AR}$ agonist reactivates BAT and markedly improved glucose homeostasis. NASH is a manifestation of hepatic lipotoxicity in which insulin resistance is a key player. Hence, many studies have investigated whether insulin sensitizers could be a therapeutic option for NASH. Treatment with metformin was not shown to be superior to placebo in ameliorating liver histology in patients with NAFLD [24-26]. Also, while glitazones markedly improve insulin sensitivity, their effects on liver pathology are marginal [27]. Here, we confirmed that improvement of glucose homeostasis alone obtained by $\beta 3 \mathrm{AR}$ agonistdriven BAT activation is not associated with reversal of liver complications, albeit improving steatosis. This adds to the growing evidence that improving insulin sensitivity alone has no therapeutic benefit in NASH management.

Many studies support the importance of lifestyle changes, especially weight loss, on NASH $[3,4,19]$. Therefore, 
A

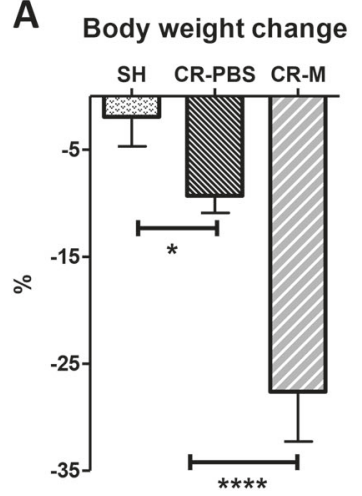

C

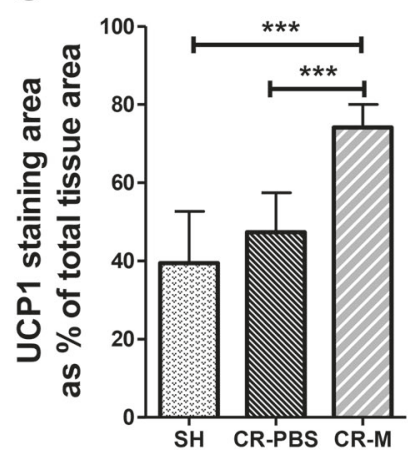

B

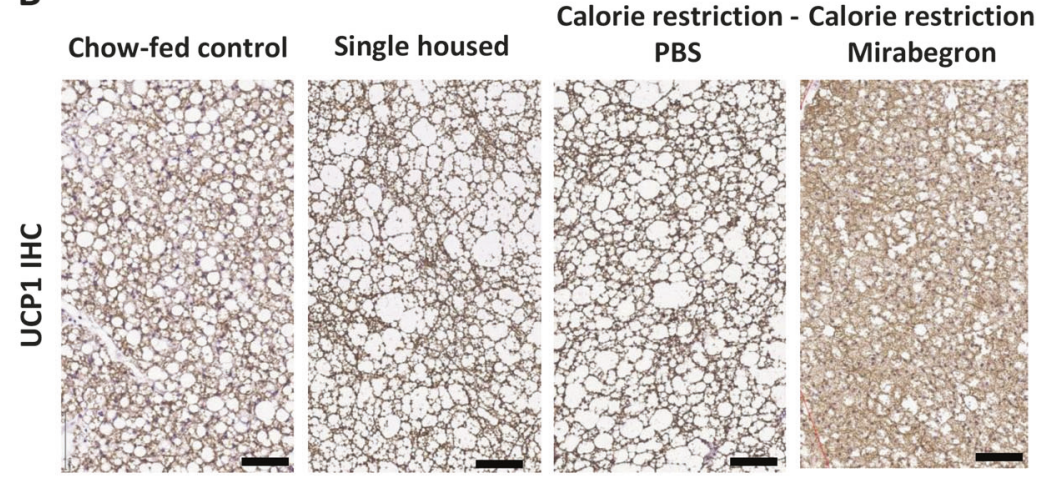

D
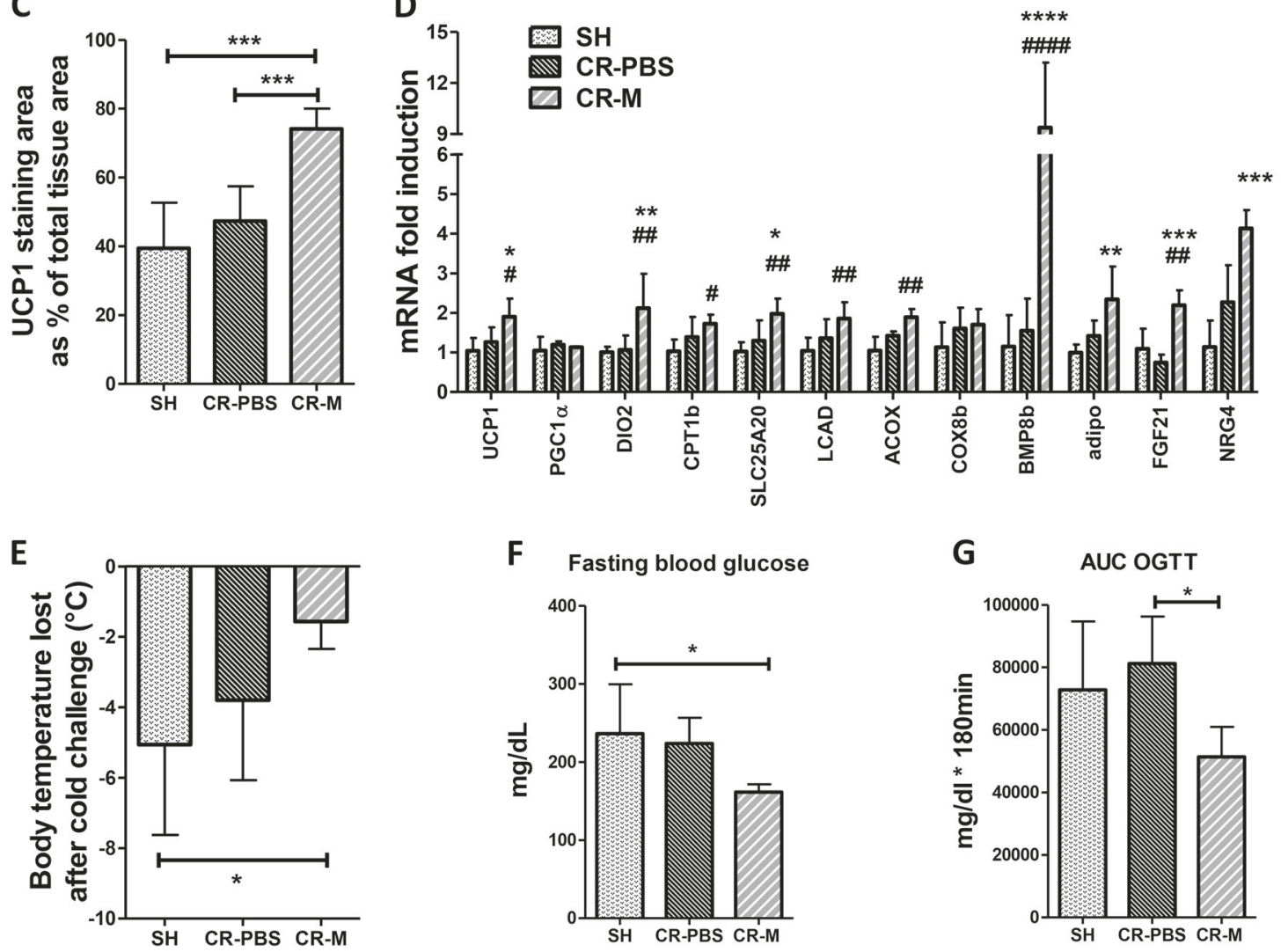

F Fasting blood glucose

G

AUC OGTT
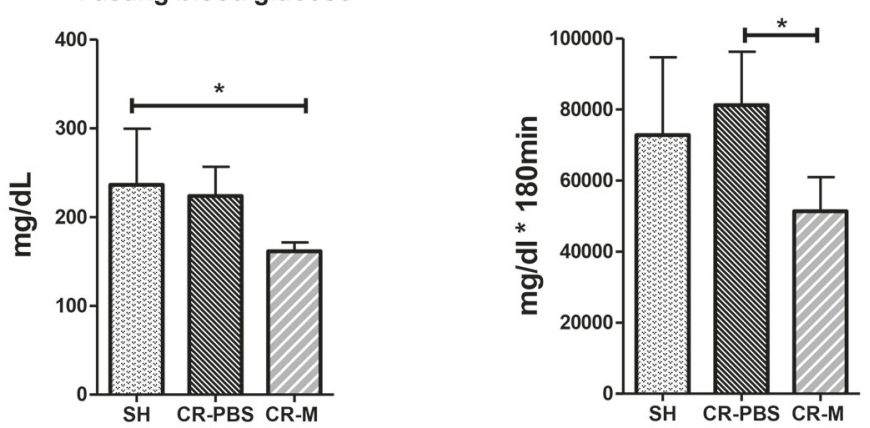

Fig. 5 Effect of caloric restriction and mirabegron treatment on metabolic parameters and BAT. After 8 weeks of HFD ad libitum, fozl foz mice were single-housed and randomized on the basis of body weight and fasting glycemia in three groups receiving either the HFD ad libitum or a daily injection of vehicle (single-housed, $\mathrm{SH}, n=5$ ), $2.5 \mathrm{~g}$ of HFD a day and a daily injection of vehicle (caloric restriction -PBS; CR-PBS, $n=5$ ), or $2.5 \mathrm{~g}$ of HFD a day and a daily injection of mirabegron $(10 \mathrm{mg} / \mathrm{kg} /$ day) $(\mathrm{CR}-\mathrm{M}, n=6)$ for 4 weeks. a Body weight change during the 4-week treatment period; (b) immunohistochemical staining of UCP1 in BAT (bar size, $100 \mu \mathrm{m}$ ); (c) morphometrical quantification of UCP1 immunostaining; and d mRNA

weight loss through lifestyle changes is the first-line, effective therapy for NASH. The major limitation of weight loss therapy is the difficulty to maintain weight loss over time. Interestingly in our study, when coupled with caloric restriction, BAT stimulation led to additional weight loss, expression of genes related to thermogenesis, $\beta$-oxidation, and batokines in BAT. Data are expressed as fold induction compared to values in ad libitum-fed, PBS-treated, and single-housed control group; (e) change in intrarectal temperature before and after a 2-h exposure at $4^{\circ}$ C; (f) fasting blood glucose levels; and (g) area under the glucose curves of the glucose tolerance test performed at the end of the treatment period. Data are mean \pm SEM. One-way ANOVA followed by post hoc Bonferroni analysis. ${ }^{*} p<0.05 ;{ }^{* *} p<0.01 ;{ }^{* * *} p<0.001$; in

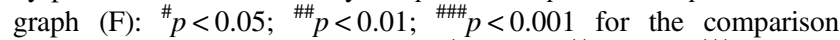
between SH controls and CR-M and ${ }^{*} p<0.05 ;{ }^{* *} p<0.01 ;{ }^{* * *} p<0.001$ for the comparison between CR-PBS and CR-M

thus supporting increased BAT thermogenesis as an antiobesity adjuvant option. Cumulatively, the benefit of mirabegron treatment over food restriction alone on steatosis, ballooning, and inflammation achieved a reversal of NASH. 
Fig. 6 Effect of calorie restriction and mirabegron treatment on the liver histology. After 8 weeks of HFD ad libitum, foz/foz mice were single-housed and randomized on the basis of body weight and fasting glycemia in three groups receiving either the HFD ad libitum or a daily injection of vehicle (single-housed, $\mathrm{SH}, n=$ 5), $2.5 \mathrm{~g}$ of HFD a day and a daily injection of vehicle (calorie restriction-PBS; CR-PBS, $n=$ 5 ), or $2.5 \mathrm{~g}$ of HFD a day and a daily injection of mirabegron (10 mg/kg/day) (CR-M, $n=6$ ). Liver tissues were analyzed after 4 weeks. Foz/foz mice on a normal diet (chow fed) for the same 12-week duration are controls. a Hematoxylin and eosin staining of liver sections (original magnification, 10x upper panels, $20 \times$ lower panels), (b) F4/80 immunostaining for macrophages, and c Ly6G immunostaining for neutrophils. In $\mathbf{c}$ arrows point to isolated neutrophils, while neutrophil aggregates are well identifiable. Bar size, $100 \mu \mathrm{m}$
A
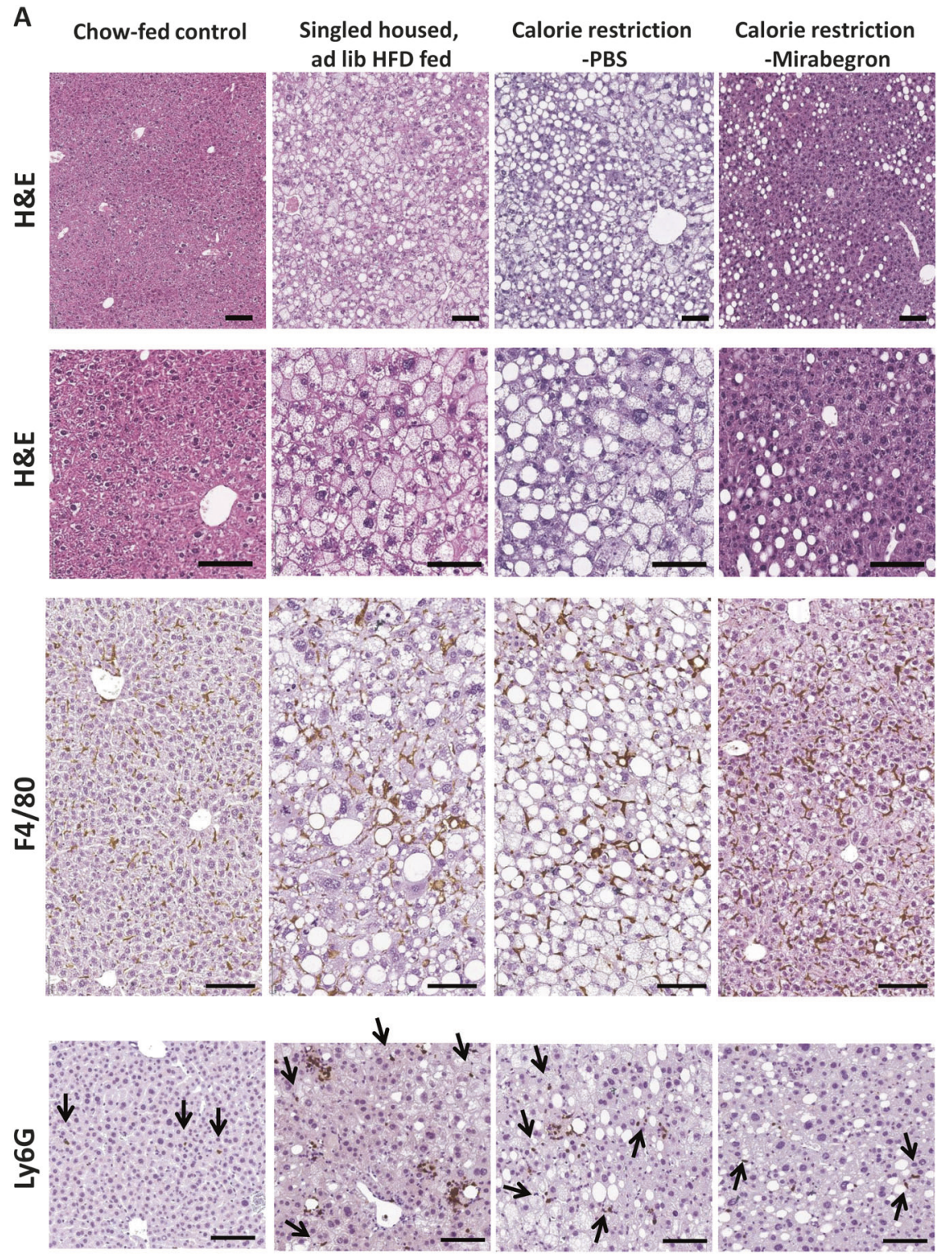

Is NASH reversal all due to the magnitude of weight loss? An intensive weight loss program for 1 year in patients with NASH led to resolution of NASH according to the percentage of weight loss achieved: 26,64 , and $90 \%$ of patients who lost between 5 and $7 \%, 7-10 \%$, and $>10 \%$ of their starting BW resolved NASH [4]. In clinical series using bariatric surgery, NASH resolution correlates with the amount of weight loss after surgery [28]. Literature in animal models of NASH provides little data except for one study: Larter et al. performed an experiment in which HFDfed fozlfoz mice were reverted to a low-fat rodent chow for 12 weeks. Food/calorie consumption was not reported. The regimen attenuated hepatic inflammation and steatosis but did not significantly reduce body weight $(-12 \%)$ and hepatocellular injury [15]. In our hands, quantitative but not qualitative caloric restriction alone that achieved a $10 \%$ weight loss also alleviated molecular inflammatory signature, but not steatosis or hepatocellular injury. For ethical reasons, we could not restrict food intake further such as to obtain a $25 \%$ body weight loss matching weight loss achieved with moderate food restriction and BAT activation to answer this question.

Previous reports by us [11] and others $[9,29,30]$ indicate that increasing thermogenic capacities prevents liver steatosis in $o b / o b$ and in HFD-fed wild-type or foz/foz mice. Here again, we show that in animals with preestablished $\mathrm{NASH}$, activation of the thermogenic program reduces lipogenesis and significantly limits the accumulation of lipids in the liver. Moderate food restriction causing a $10 \%$ weight loss had no such anti-lipogenic effect. A first explanation may involve a shift of lipid flux away from the liver to be used as substrates by the activated BAT [31]. 
A

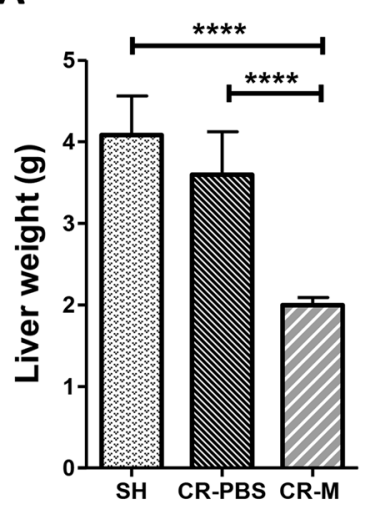

B

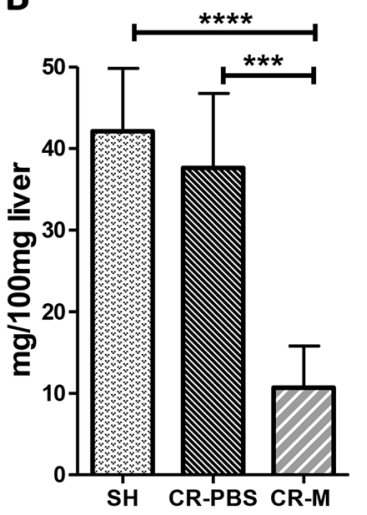

C

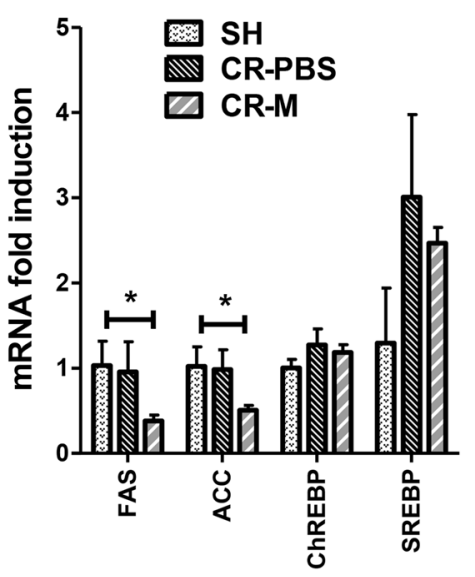

D

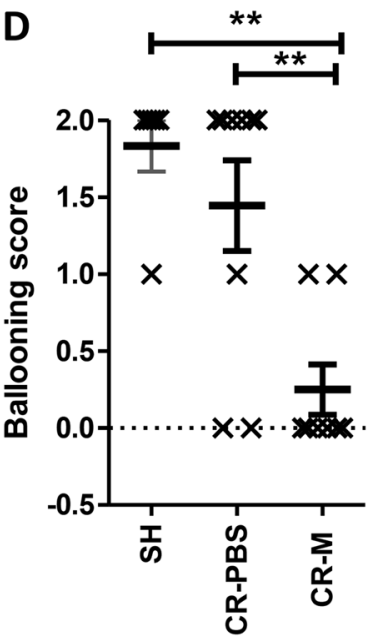

E

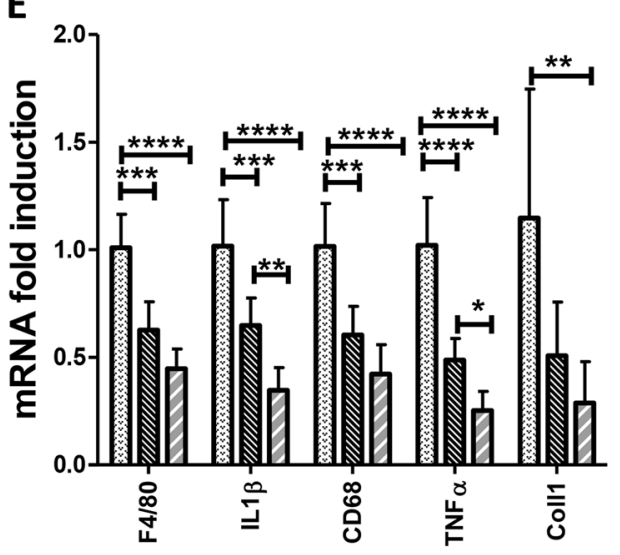

$\mathbf{F}$

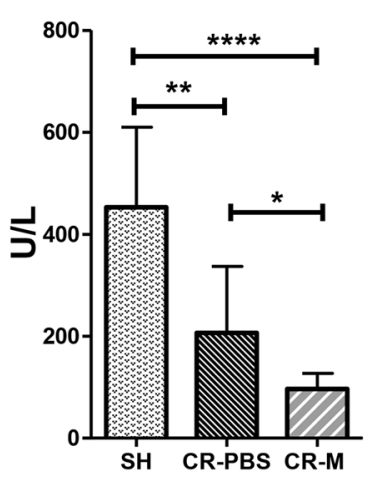

G

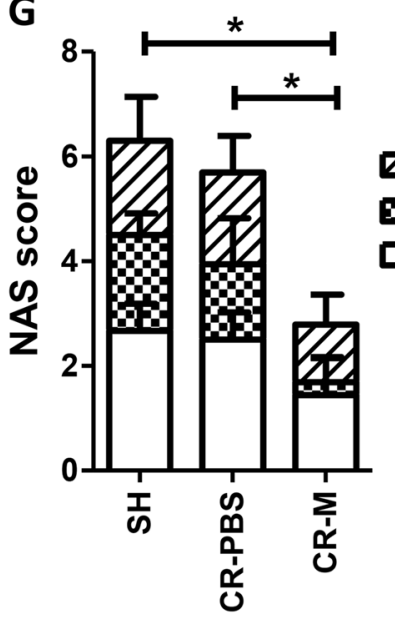

Z7 inflammation ballooning steatosis
Fig. 7 Calorie restriction coupled to mirabegron treatment improves NASH. a Liver weight; (b) liver lipid content; and c mRNA expression of genes related to lipogenesis. Data are expressed as fold induction compared to values of ad libitum-fed, PBS-treated, and single-housed control group; (d) ballooning score as per Kleiner et al. [39]; (e) mRNA expression of inflammatory genes. Data are expressed as fold induction compared to values of ad libitum-fed, PBS-treated,

Indeed, $\beta 3 \mathrm{AR}$ treatment increased the expression of LPL, the rate-limiting enzyme for uptake of FFA in BAT, as well as it activates the enzymatic machinery for $\beta$-oxidation and uncoupling. It also increased glucose uptake (via GLUT4 upregulation) and consumption, retrieving glucose as a substrate for hepatic lipogenesis. Interestingly, in our experiments, treatment to activate BAT causes a downregulation of hepatic lipogenic genes, while the $\beta$-oxidative pathway for fat burning remained unchanged. This also occurs upon acute (3 days) administration of mirabegron that activates BAT in chow-fed mice (see Suppl. Figure 4). Hepatocytes do not express $\beta 3$ adrenoreceptor, therefore a direct effect is unlikely [32]. Another hypothesis is that BAT activation indirectly decreases lipogenesis and liver lipid storage through secretion of endocrine factors. NRG4, highly enriched in BAT, is a secreted protein which, upon and single-housed control group; (f) serum alanine aminotransferases levels; (g) histological NAS score [39] within each group of the mean steatosis score (white part of the bars), the ballooning score (dotted part of the bars), and the inflammatory score (hatched part of the bars). Data are represented as mean \pm S.E.M. One-way ANOVA followed by post hoc Bonferroni analysis ${ }^{*} p<0.05 ;{ }^{* *} p<0.01 ;{ }^{* * *} p<0.001$

binding to liver receptor, regulates de novo liver lipid synthesis [17]. In humans, NRG4 inversely correlates with liver fat mass [17]. In our experiments using two different $\beta 3 \mathrm{AR}$ agonists (CL-316,243 and mirabegron), decreased hepatic lipogenesis was associated with increased NRG4 transcript and protein (Suppl. Figure 6) in BAT of fozlfoz mice. Circulating NRG4 levels, which would support the distant effect, were, however, not quantifiable using ELISA tests available. Besides NRG4, other batokines could also positively tune energy homeostasis. For example, engrafted BAT sustains the secretion of FGF21 and adiponectin, two endocrine factors with metabolic regulatory effects $[9,22]$. In our study, CL-316,243 induced FGF21 in BAT by a factor 3 in mice with preestablished NASH but this effect was less in mice under calorie restriction and mirabegron treatment. Further studies will be required to confirm 
whether and which endocrine factors produced as part of the thermogenic program can act as a negative regulator of lipogenesis in the obese and dysmetabolic context.

In addition to decreased hepatic lipid content, we noted that $\beta 3 \mathrm{AR}$ agonists (whether CL-316,243 or mirabegron) lessened hepatocyte ballooning, a likely manifestation of lipotoxicity. Clearance of ballooned hepatocytes was especially noticeable in mice under calorie restriction and mirabegron treatment, a therapeutic regimen particularly efficient in reducing steatosis, suggesting that lipid clearance is key to alleviate lipotoxicity.

One of the very interesting findings in the present study is that reduction in quantity (but not quality) of calorie intake for a limited period switches the molecular signature of inflammation in the liver. Food restriction alone significantly decreased F4/80 and CD68 transcripts, and proinflammatory cytokines such as TNF or IL1 $\beta$, lessened aggregation of inflammatory macrophages and recruitment on neutrophils. Similarly, Larter et al. reported a less inflammatory profile in fozlfoz mice raised on a HFD and then switched to a carbohydrate- rich-fat-poor standard rodent chow [15]. Addition of a $\beta 3 \mathrm{AR}$ agonist to mice subjected to food restriction achieved a significant antiinflammatory effect in the liver. By contrast, BAT activation alone had no significant impact on hepatic inflammation, as shown in HFD-fed foz/foz mice or MCD-fed $d b / d b$ mice treated with a $\beta 3 \mathrm{AR}$ agonist.

It is now certain that adult humans have appreciable amounts of thermogenic adipose tissue [6, 31-33]. Obesity associates with decreased functional BAT [32] and functional studies performed in a limited number of patients revealed a low-BAT activity in subjects with a fatty liver [34]. At this stage, it remains unclear as to whether sufficient amounts of active thermogenic adipose tissue are present in NAFLD/NASH patients to exert beneficial therapeutic effects, even when fully activated. There is a tremendous interest in finding strategies to increase the mass and the activity of thermogenic adipose tissue. So far, few clinical trials reported the possibility of stimulating BAT activity in healthy adults through cold exposure or with the B3AR agonist mirabegron. These short-term studies associated increased BAT activity with an increased resting metabolic rate, whole-body lipolysis, and adipose tissue insulin sensitivity, but not obesity [34, 35, 36-38]. Longterm studies in obese subjects, necessary to evaluate the impact on body weight, energy balance, and metabolic syndrome, are not (yet) available. In light of our results, it will be interesting to assess BAT and thermogenic capacity in patients with NASH and to test, in those with responsive tissue, if activation of the BAT function helps maintaining body weight loss, and brings an additional long-term therapeutic benefit on NASH over lifestyle change program.
In summary, $\beta 3 \mathrm{AR}$ agonist treatment increases adaptive nonshivering thermogenesis, consumption of energy substrates, and improves glucose tolerance in morbidly obese mice with metabolic syndrome. While it alleviates partly hepatic fat accumulation and hepatocellular injury, $\beta 3 \mathrm{AR}$ agonist treatment alone did not reverse NASH. When coupled with weight loss therapy (here a caloric restriction), $\beta 3 \mathrm{AR}$ agonist treatment significantly contributes to a negative energy balance and improves both metabolic syndrome and NASH. This study provides insights for the potential use of BAT stimulation as an adjuvant therapy for human NASH.

Acknowledgments The authors thank Natacha Feza-Bingi (UCL, Brussels, Belgium) and Mathilde Beka (UCL, Brussels, Belgium) for animal breeding, genotyping, and care; and Anne Bol (UCL, Brussels, Belgium) for PET/CT imaging. The work was financially supported by "Communauté française de Belgique - Actions de Recherche Concertées" (12/17-047) and unrestricted grants from Bristol-Myers Squibb Belgium, MSD Belgium, Gilead Belgium, Janssen Pharmaceutica Belgium, and Abbvie Belgium.

Competing interests The authors have no conflict of interest in relation to this work to disclose.

\section{References}

1. LaBrecque DR, Abbas Z, Anania F, et al. World Gastroenterology Organisation global guidelines: nonalcoholic fatty liver disease and nonalcoholic steatohepatitis. J Clin Gastroenterol. 2014;48:467-73.

2. Vernon G, Baranova A, Younossi ZM. Systematic review: the epidemiology and natural history of nonalcoholic fatty liver disease and nonalcoholic steatohepatitis in adults. Aliment Pharmacol Ther. 2011;34:274-85.

3. Chalasani N, Younossi Z, Lavine JE, et al. The diagnosis and management of nonalcoholic fatty liver disease: Practice Guideline by the American Association for the Study of Liver Diseases, American College of Gastroenterology, and the American Gastroenterological Association. Hepatology. 2012;55:2005-23.

4. Vilar-Gomez E, Martinez-Perez Y, Calzadilla-Bertot L, et al. Weight loss through lifestyle modification significantly reduces features of nonalcoholic steatohepatitis. Gastroenterology. 2015; 149:367-78.e5.

5. Poekes L, Lanthier N, Leclercq IA. Brown adipose tissue: a potential target in the fight against obesity and the metabolic syndrome. Clin Sci. 2015;129:933-49.

6. Virtanen KA, Lidell ME, Orava J, et al. Functional brown adipose tissue in healthy adults. N Engl J Med. 2009;360:1518-25.

7. van Marken Lichtenbelt WD, Schrauwen P. Implications of nonshivering thermogenesis for energy balance regulation in humans. AJP Regul Integr Comp Physiol. 2011;301:R285-96.

8. Leitner BP, Huang S, Brychta RJ, Duckworth CJ, Baskin AS, McGehee S, et al. Mapping of human brown adipose tissue in lean and obese young men. Proc Natl Acad Sci. 2017;114:8649-54.

9. Liu X, Wang S, You Y, et al. Brown adipose tissue transplantation reverses obesity in $\mathrm{Ob} / \mathrm{Ob}$ mice. Endocrinology. 2015;156: 2461-9.

10. Yuan X, Wei G, You Y, et al. Rutin ameliorates obesity through brown fat activation. FASEB J. 2017;31:333-45.

11. Poekes L, Legry V, Schakman O, et al. Defective adaptive thermogenesis contributes to metabolic syndrome and liver steatosis in obese mice. Clin Sci. 2017;131:285-96. 
12. Larter CZ, Yeh MM, Van Rooyen DM, et al. Roles of adipose restriction and metabolic factors in progression of steatosis to steatohepatitis in obese, diabetic mice. J Gastroenterol Hepatol. 2009;24:1658-68.

13. Arsov T, Silva DG, O’Bryan MK, et al. Fat aussie-a new Alström syndrome mouse showing a critical role for ALMS1 in obesity, diabetes, and spermatogenesis. Mol Endocrinol. 2006; 20:1610-22.

14. Legry V, Van Rooyen DM, Lambert B, et al. Endoplasmic reticulum stress does not contribute to steatohepatitis in obese and insulin-resistant high-fat-diet-fed foz/foz mice. Clin Sci. 2014;127:507-18.

15. Larter CZ, Yeh MM, Haigh WG, et al. Dietary modification dampens liver inflammation and fibrosis in obesity-related fatty liver disease. Obesity. 2013;21:1189-99.

16. Liu J, Xu Y, Hu Y, Wang G. The role of fibroblast growth factor 21 in the pathogenesis of nonalcoholic fatty liver disease and implications for therapy. Metabolism. 2015;64:380-90.

17. Wang GX, Zhao XY, Meng ZX, et al. The brown fat-enriched secreted factor $\mathrm{Nrg} 4$ preserves metabolic homeostasis through attenuation of hepatic lipogenesis. Nat Med. 2014;20:1436-43.

18. Leclercq IA, Lebrun VA, Stärkel P, Horsmans YJ. Intrahepatic insulin resistance in a murine model of steatohepatitis: effect of PPAR $\gamma$ agonist pioglitazone. Lab Invest. 2007;87:56-65.

19. Romero-Gómez M, Zelber-Sagi S, Trenell M. Treatment of NAFLD with diet, physical activity and exercise. J Hepatol. 2017;67:829-46.

20. Marchesini G, Mazzella N, Forlani G. Weight loss for a healthy liver. Gastroenterology. 2015;149:274-8.

21. Calmasini FB, de Oliveira MG, Alexandre EC. Long-term treatment with the beta-3 adrenoceptor agonist, mirabegron ameliorates detrusor overactivity and restores cyclic adenosine monophosphate (cAMP) levels in obese mice. Neurourol Urodyn. 2017;36:1511-8.

22. Stanford KI, Middelbeek RJ, Townsend KL, et al. Brown adipose tissue regulates glucose homeostasis and insulin sensitivity. J Clin Invest. 2013;123:215-23.

23. Hepler C, Shao M, Xia JY, et al. Directing visceral white adipocyte precursors to a thermogenic adipocyte fate improves insulin sensitivity in obese mice. eLife. 2017;6:1-33.

24. Haukeland JW, Konopski Z, Eggesb $\emptyset$ HB, et al. Metformin in patients with nonalcoholic fatty liver disease: a randomized, controlled trial. Scand J Gastroenterol. 2009;44:853-60.

25. Li Y, Liu L, Wang B, et al. Metformin in nonalcoholic fatty liver disease: a systematic review and meta-analysis. Biomed Rep. 2013;1(1):57-64
26. Shields WW, Thompson KE, Grice GA, et al. The effect of metformin and standard therapy versus standard therapy alone in nondiabetic patients with insulin resistance and nonalcoholic steatohepatitis (NASH): a Pilot Trial. Ther Adv Gastroenterol. 2009;2:157-63.

27. Sanyal AJ, Chalasani N, Kowdley KV, et al. Pioglitazone, vitamin E, or placebo for nonalcoholic steatohepatitis. N Engl J Med. 2010;362:1675-85.

28. Lassailly G, Caiazzo R, Buob D, et al. Bariatric surgery reduces features of nonalcoholic steatohepatitis in morbidly obese patients. Gastroenterology. 2015;149:379-88.

29. Liu X, Zheng Z, Zhu X, et al. Brown adipose tissue transplantation improves whole-body energy metabolism. Cell Res. 2013;23:851-4.

30. Wang H, Liu L, Lin JZ, et al. Browning of white adipose tissue with roscovitine induces a distinct population of UCP1+ adipocytes. Cell Metab. 2016;24:835-47.

31. Bartelt A, Bruns OT, Reimer R, et al. Brown adipose tissue activity controls triglyceride clearance. Nat Med. 2011;17:200-6.

32. Ghosh PM, Shu Z-J, Zhu B, et al. Role of b-adrenergic receptors in regulation of hepatic fat accumulation during aging. J Endocrinol. 2012;213:251-61.

33. Cypess AM, White AP, Vernochet C, et al. Anatomical localization, gene expression profiling, and functional characterization of adult human neck brown fat. Nat Med. 2013;19:635-9.

34. Yilmaz Y, Ones T, Purnak T, et al. Association between the presence of brown adipose tissue and nonalcoholic fatty liver disease in adult humans. Aliment Pharmacol Ther. 2011;34:318-23.

35. Chondronikola M, Volpi E, Børsheim E, et al. Brown adipose tissue activation is linked to distinct dystemic dffects on lipid metabolism in humans. Cell Metab. 2016;23:1200-6.

36. Cypess AM, Weiner LS, Roberts-toler C, et al. Activation of human brown adipose tissue by a $\beta 3$-adrenergic receptor agonist. Cell Metab. 2016;21:33-8.

37. Hibi M, Oishi S, Matsushita M, et al. Brown adipose tissue is involved in diet-induced thermogenesis and whole-body fat utilization in healthy humans. Int J Obes. 2016;40:1655-61.

38. Matsushita M, Yoneshiro T, Aita S, et al. Impact of brown adipose tissue on body fatness and glucose metabolism in healthy humans. Int J Obes. 2014;38:812-7.

39. Kleiner DE, Brunt EM, Van Natta M, et al. Design and validation of a histological scoring system for nonalcoholic fatty liver disease. Hepatology. 2005;41:1313-21.

40. Andrew J. Whittle, Stefania Carobbio, Luís Martins, et al. BMP8B Increases Brown Adipose Tissue Thermogenesis through Both Central and Peripheral Actions. Cell 2012;149:871-885. 